\title{
Control de las ruedas delanteras de robots móviles
}

Fausto Freire Carrera

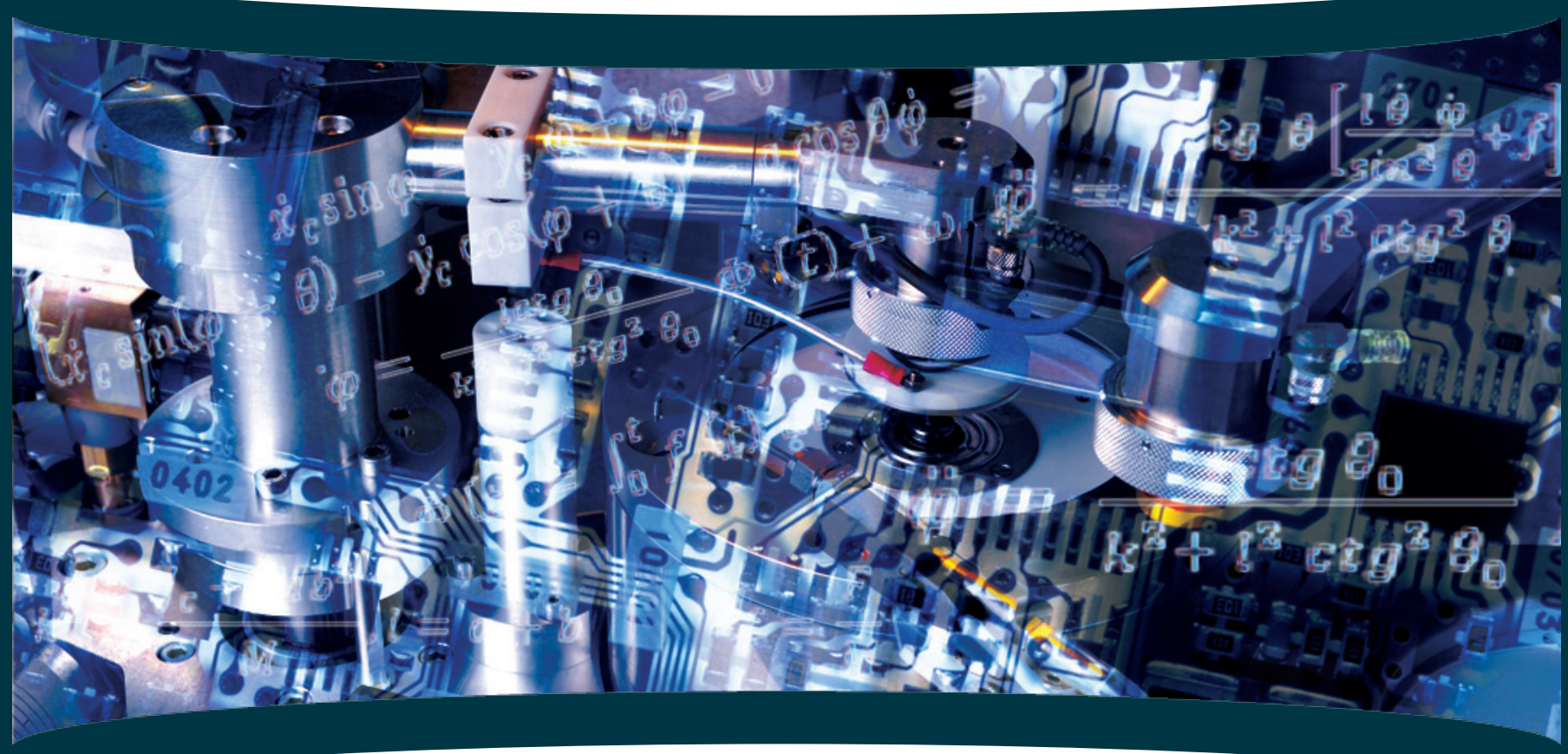

Resumen-El presente artículo trata sobre un modelo para controlar el movimiento de las ruedas delanteras de los robots móviles, ya que al ser manipulados remotamente, es necesario de antemano conocer su posible comportamiento, los mejores resultados de control se obtuvieron mediante la utilización de controladores difusos.

Palabras clave-Control difuso, internet, robots, ruedas.

La Tele-robótica como una rama aplicada del conocimiento, es la que reúne todas las características mínimas necesarias al momento de sustituir, la presencia de personas en lugares de trabajo peligrosos, hostiles y de poca accesibilidad, permitiendo alcanzar mejores resultados consiguiendo una importante economía de recursos físicos, económicos y de tiempo (Jatsun, Freire, Dyshenko, Shadrina, 2005, 2006).

El control de robots móviles se lo viene realizando desde diversas perspectivas y consideraciones, a continuación se plantea un modelo que permite realizar el control del

1Fausto Freire Carrera, Coordinador de Investigación de la Dirección General de Posgrados, 095800212, ffreire@ute.edu.ec movimiento de un robot móvil de ruedas remotamente, utilizando como medio de comunicación la red de ordenadores Internet (Fig.1)
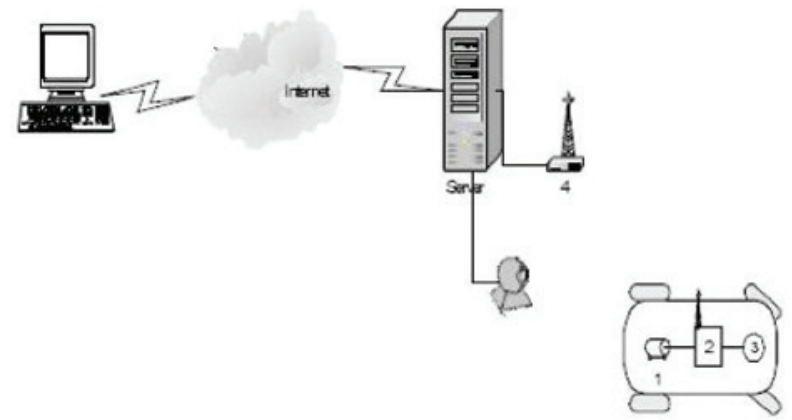

Figura 1. Modelo de control del robot móvil a través de la red Internet.

En este modelo se presentan los elementos mínimos indispensables para realizar el control de un robot móvil desde el Internet, compuesto por las interfaces entre el operador y el 
servidor del robot y la cámara.

Un posible modelo matemático que permite analizar la dinámica del movimiento de las ruedas delanteras del móvil en función de fuerzas (Fig. 2) se presenta a continuación, considerando al móvil como un sistema mecánico simplificado compuesto por dos cuerpos (modelo de Lineikin), el primer cuerpo está compuesto por el puente delantero $A_{1} A_{2}$, y las ruedas $A_{1}$ y $A_{2}$, el segundo cuerpo se encuentra formado por la plataforma y el puente trasero $\mathrm{B}_{1} \mathrm{BB}_{2}$

El movimiento se realiza mediante la aplicación de fuerzas externas que actúan sobre el punto B y por la fricción con la superficie de contacto.

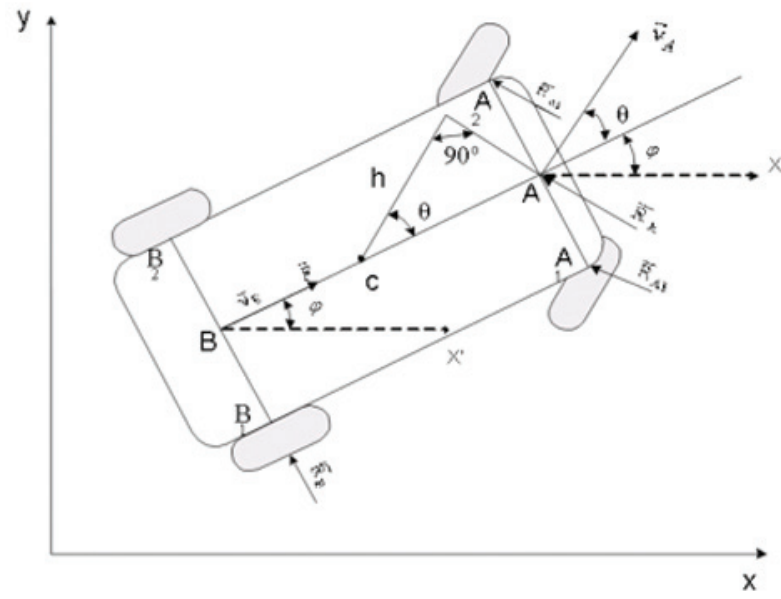

Figura 2. Esquema del modelo del robot móvil.

Considerando el movimiento del robot móvil en condiciones ideales, las ecuaciones cinemáticas de los enlaces del robot móvil de la figura 2, está representado mediante las siguientes ecuaciones (1)

$$
\left\{\begin{array}{c}
\dot{x}_{c} \sin \varphi-\dot{y}_{c} \varphi+b \varphi=0 \\
\dot{x}_{c} \sin (\varphi+\theta)-\dot{y}_{c} \cos (\varphi+\theta)-a \cos \theta \dot{\phi}=0
\end{array}\right.
$$

Donde:

$A C=a, C B=b$

En vista de las fuerzas que actúan sobre el robot, las ecuaciones (1) tienen el aspecto de las ecuaciones dinámicas de movimiento con multiplicadores indefinidos de Lagranian $\lambda 1, \lambda 2$ para los sistemas mecánicos con enlaces lineales ideales no Holonomos, entonces obtenemos el sistema de las ecuaciones dinámicas diferenciales del movimiento del robot de la siguiente manera:

$$
\left\{\begin{array}{l}
M \ddot{x}_{c}=F \cos \varphi+\lambda_{1} \sin \varphi+\lambda_{2} \sin (\varphi+\theta) \\
M \ddot{y}_{c}=F \sin \varphi-\lambda_{1} \cos \varphi-\lambda_{2} \cos (\varphi+\theta) \\
J_{c} \ddot{\varphi}=\lambda_{1} b-\lambda_{2} a \cos \theta
\end{array}\right.
$$

Resolviendo el sistema de ecuaciones dinámicas diferenciales (2) y cinemáticas de los enlaces (1) en función de cinco incógnitas $\dot{x}_{c}, y_{c}, \lambda_{1}, \lambda_{2}, \varphi_{\text {r }}$ se obtiene la ecuación (3) que permite de forma compacta resolver el sistema (2):

$$
\ddot{\varphi}=\frac{\operatorname{lctg} \theta\left[\frac{l \dot{\theta} \dot{\phi}}{\sin ^{2} \theta}+f\right]}{k^{2}+l^{2} \operatorname{ctg}^{2} \theta}
$$

Donde:

$$
k^{2}=\frac{J_{c}+M b^{2}}{M}, l=a+b \quad f=\frac{F}{M}
$$

La magnitud $\mathrm{f}$ representa la fuerza atribuida a la unidad de la masa, y el coeficiente $k$ es el radio de la inercia del robot acerca del eje vertical que pasa a través del punto n, $\mathrm{M}$ - la masa de todo el sistema, Jc - momento de la inercia del sistema cerca del centro de las masas.

La solución gráfica de la ecuación (3) con la consideración k=1 y I=1 es la siguiente (Fig. 3, 4).
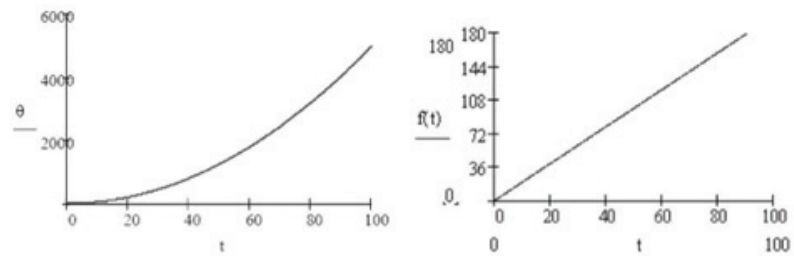

Figura 3. Gráficos de la dirección del movimiento de las ruedas delanteras y la fuerza que actúa sobre el robot.

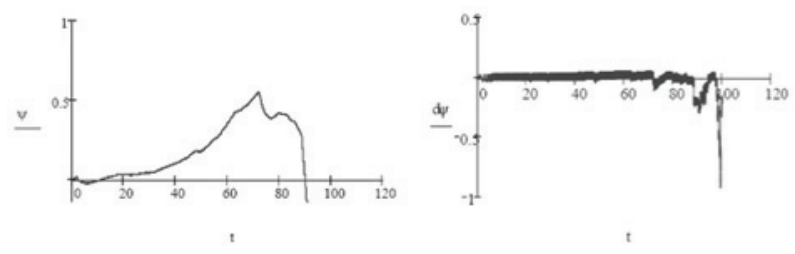

Figura 4. Gráficos del ángulo de giro $(\Psi)$ y la velocidad angular de giro del eje $A B$ del robot.

Uno de los modos más simples para resolver el 
sistema de ecuaciones diferenciales (3) cuando se tiene coeficientes variables (dinámicos) es mediante el método de la congelación de coeficientes. "Son congelados" en este caso el valor de la variable $\Psi$ que conduce a la congelación de los coeficientes de la ecuación diferencial (3).

En este caso el sistema con coeficientes variables es reducido a un sistema con los parámetros constantes que permite aplicar, los métodos conocidos de resolución de sistemas de ecuaciones (Lure, Eriat, 2004).
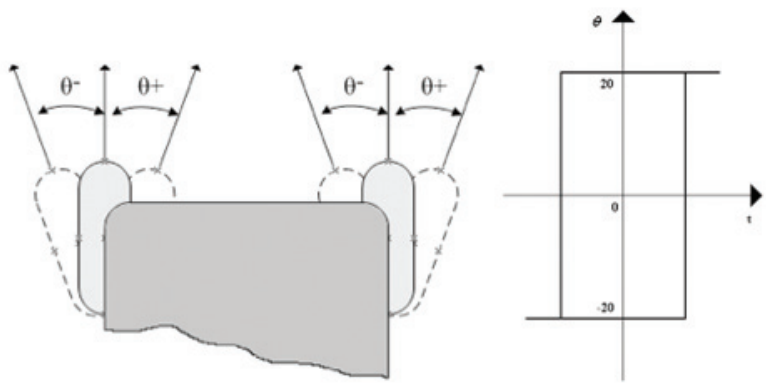

Figura 5. Ámbito de movimiento de las ruedas delanteras

Las ruedas delanteras del robot móvil giran en un intervalo definido entre $-20 \leq \theta \leq 20$ y fijamos el sistema en los puntos $\theta=-20, \theta=-10, \theta=0, \theta=10, \theta=20$, de esta forma en vez de una ecuación con coeficientes variables tenemos cinco, con coeficientes fijos (Fig. 5). Consideramos que el control del volante del móvil permite conservar un valor constante del ángulo $\theta$, es decir $\theta=0^{\circ} \theta \theta=\theta 0$, el valor de $f$ puede ser cualquier función del tiempo, entonces la ecuación del objeto (3) tendrá el siguiente aspecto.

$$
\ddot{\varphi}=\frac{\operatorname{lotg} \theta_{0}}{k^{2}+l^{2} \operatorname{ctg}^{2} \theta_{0}} f(t)
$$

Donde, $\varphi$ y $\Phi$ son las variables de control de entrada y salida del sistema, de (4) obtenemos la velocidad angular de movimiento.

$$
\begin{gathered}
\varphi=\frac{\operatorname{lotg} \theta_{0}}{k^{2}+l^{2} \operatorname{ctg}^{2} \theta_{0}} \Phi(t)+\omega_{0} \\
\Phi(t)=\int_{0}^{t} f(t) d t
\end{gathered}
$$

Es decir la función $\Phi(\mathrm{t})$ después de multiplicar con la masa $\mathrm{M}$ determina el impulso completo de las fuerzas $\mathrm{F}$ durante el movimiento desde el inicio del movimiento hasta el momento t.

El sistema de control automático del robot móvil controlado desde el Internet con la utilización de controladores difusos (Jan, 1998) y teniendo en consideración las restricciones impuestas antes, como también considerando $\varphi$ y $\Phi$ las variables de salida y entrada del sistema tenemos lo siguiente (Smirnov, Hasanova, 2003), (Bao-Gang, otros, 2001).

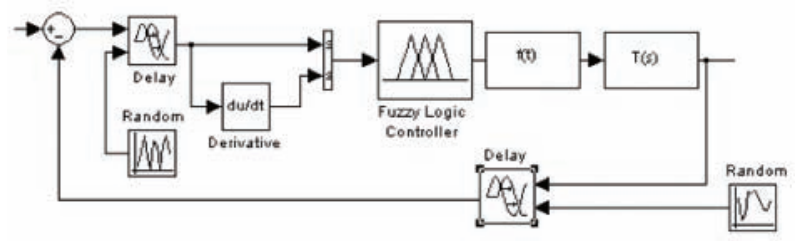

Figura 6. SCA con controlador difuso

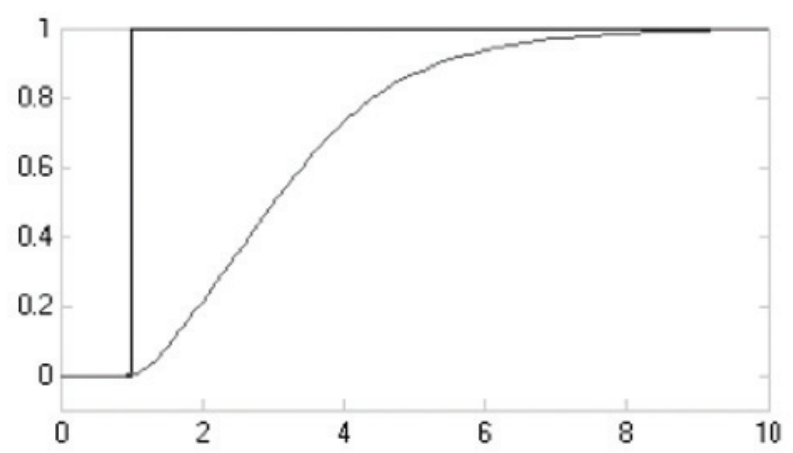

Figura 7. Reacción del sistema a la señal escalón.

El control del robot móvil mediante la red Internet se reduce básicamente, a la resolución de dos problemas principales. En este caso se utilizó el siguiente esquema:

- El primer problema consiste en los mecanismos para la determinación de la posición del robot.

- El segundo problema consiste en la ejecución por parte del robot de los comandos generados por el operador.

La acción de Internet sobre el sistema está modelado mediante la generación de un valor randómico que es enviado a un bloque Delay encargado de retardar la señal en esta magnitud, tanto desde el operador hacia el robot como viceversa.

T(s) es la representación de la ecuación (5) utilizando operadores de la Plaza, el sistema reacciona a un comando de 
giro de las ruedas delanteras de 200, se utilizó un controlador difuso tipo PD, que permitió alcanzar niveles de reacción del sistema aceptables, como parámetros de control (Figura 7).

\section{Bibliografía:}

- Jatsun S.F., Freire F. C., Dyshenko V.S., Shadrina O. A., (2005). Control de Robots móviles a través de las redes Internet, Telecomunicaciones № 3.

- Lure B.J., Eriat P. Dsh., (2004). Métodos clásicos del control automático, Sant. Peterburg.- Pág. 640.

- Modelación difusa en el ambiente de MATHLAB y fuzzyTECH. (2005). SAN PETERSBURGO, Pág. 736.

- Smirnov V.A., Hasanova A.A., (2003). Metodología de realización de sistemas de control con lógica difusa, Noticias del centro científico De Chelyabinsk, numero 4 (21).

- Bao-Gang Hu, George K. I. Mann, and Raymond G. Gosine, (2001).A Systematic Study of Fuzzy PID Controllers FunctionBased Evaluation Approach, IEEE transactions on fuzzy systems, vol. 9, No. 5, October.

- Jan J, (1998). Design Of Fuzzy Controllers, Technical University of Denmark, department of Automation, Tech. report no 98-E 864 (design), 19 Aug.

- Jatsun S.F., Freire F. C., (2006). Sistema de control de robots mediante Internet, Noticias de KSTU, 2006 №1 (16).

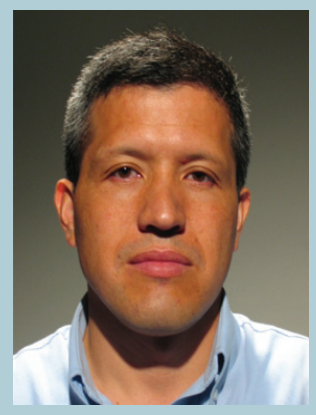

Autor: Fausto Rodrigo Freire Carrera

ffreire@ute.edu.ec

Título: Ingeniero en Sistemas.

Institución: Universidad Técnica Estatal de Kursk - UTEK (Federación de Rusia).

Facultad: Ingeniería en Sistemas, Computación y Automatización.

Título: Máster en Ciencias.

Institución: Universidad Técnica Estatal de Kursk (Federación de Rusia).

Facultad: Facultad de Ingeniería en Sistemas, Computación y Automatización.

Título: Máster en Tecnologías de la Información para la Fabricación.

Institución: Universidad Politécnica de Madrid (España).

Nombre del programa académico: Departamento de Sistemas Inteligentes Aplicados.

Título: Especialista en Robótica.

Institución: Universidad Politécnica de Madrid (España).

Nombre del programa académico: Departamento de Sistemas Inteligentes Aplicados.

Título: Ph.D en Dinámica, Resistencia, Aparatos y Dispositivos Institución: Universidad Técnica Estatal de Kursk (Federación de Rusia).

Nombre del programa académico: Facultad de Mecánica Teórica y Mecatrónica. 